\title{
Solução cardioplégica sangüínea versus acelular: avaliação hemodinâmica através de estudo experimental em cães
}

Luís Alberto DALLAN*, Sérgio Almeida de OLIVEIRA*, José Carlos R. IGLÉZIAS*, Paulo M. PÊGO-FERNANDES*, José Otávio C. AULER JÚNIOR*, Geraldo VERGINELLI*, Adib D. JATENE*

RBCCV 44205-121

DALLAN, L. A.; OLIVEIRA, S. A.; IGLÉZIAS, J. C. R.; PÊGO-FERNANDES, P. M.; AULER JÚNIOR, J. O. C.; VERGINELLI, G.; JATENE, A. D. - Soluçāo cardioplégica sangüínea versus acelular: avaliaçāo hemodinâmica através de estudo experimental em cảes. Rev. Bras. Cir. Cardiovasc., 5(3): 168$178,1990$.

RESUMO: Estudo experimental em 20 cảes permitiu avaliar hemodinamicamente o grau de proteção miocárdica à anóxia, através do emprego de soluçāo cardioplégica sangūínea e acelular. A Isquemia miocárdica foi obtida pelo pinçamento da aorta ascendente por 60 minutos, após instalação de circulação extracorpórea, seguida de reperfusāo por 120 minutos. Os animais foram divididos em quatro grupos, sendo os dois primeiros controles, mantidos em normotermia e hipotermia moderada $\left(28^{\circ} \mathrm{C}\right)$. No terceiro e quarto grupo, além de hipotermia, foram realizadas infusōes coronárias das duas soluçōes cardioplégicas. Todos os aniamis apresentaram queda do débito cardíaco (DC) aos 30 minutos de reperfusāo miocárdica. Entretanto, decorridos 120 minutos, pode-se evidenciar, nos cảes que receberam cardioplegia, a significativa recuperaçāo da pressão sistólica (PS) do VE e a não elevação da pressāo diastólica final (PDF) do VE, além da normalizaçāo do DC, o que nāo ocorreu nos grupos controle, especialmente em normotermia. No período de anóxia estudado, nāo foi possível demonstrar, com o método, diferenças estatisticamente significativas entre as soluçōes cardioplégicas.

DESCRITORES: proteçāo miocárdica, cardioplegia.

\section{INTRODUÇĀO}

O emprego de solução cardioplégica constitui recurso amplamente utilizado em cirurgia cardíaca, visando diminuir os efeitos deletérios da anóxia miocárdica.

A partir de 1972, KIRSCH et alii "reformularam os conceitos de MELROSE et alii ${ }^{12}$, reintroduzindo na prática cirúrgica 0 emprego de soluçōes que pudessem induzir à cardioplegia. Desde então, inúmeros relatos de literatura se sucederam, sugerindo substâncias que proporcionassem melhor proteçāo ao miocárdio $1,3,6,7,9,14,15$. FOLLETTE et alii ${ }^{5}$, em 1978 , descreveram vantagens na inclusăo de sangue como veículo de infusão dessas soluçōes.

Por outro lado, inúmeros métodos vêm sendo empregados na avaliaçāo miocárdica pós anóxia, especialmente morfológicos ${ }^{4} \mathrm{e}$ ultramicroscópicos 13. Acreditamos, entretanto, que o desempenho hemodinâmico do coração pós anóxia se constitua num parâmetro adequado e objetivo na avaliação da proteçāo miocárdica.

Procuramos avaliar, neste trabalho, a real con-

Trabalho realizado no Instituto do Coração do Hospital das Clínicas da Faculdade de Medicina da Universidade de Sâo Paulo. São Paulo, SP, Brasil.

Recebido para publicação em 18 de setembro de 1990.

* Do Instituto do Coraçáo do Hospital das Clínicas da Faculdade de Medicina da Universidade de São Paulo.

Endereço para separatas: Luís Alberto Dallan. Av. Dr. Enéas de Carvalho Aguiar, 44. Divisão Cirúrgica. 05403 São Paulo. SP. Brasil. 
DALLAN, L. A.; OLIVEIRA, S. A.; IGLÉZIAS, J. C. R.; PÊGO-FERNANDES, P. M.; AULER JÚNIOR, J. O. C.; VERGINELLI, G.; JATENE, A. D. - Solução cardioplégica sangūínea versus acelular: avaliação hemodinâmica através de estudo experimental em cães. Rev. Bras. Cir. Cardiovasc., 5(3): 168-178, 1990.

tribuiçāo do emprego de sangue autólogo como substrato básico da solução cardioplégica, através de estudo experimental em cães.

\section{CASUÍSTICA E MÉTODOS}

Foram estudados, na Divisão de Experimentação do Instituto do Coração do Hospital das Clínicas da Faculdade de Medicina da Universidade de São Paulo, 20 cães mestiços, de ambos os sexos, com pesos variáveis de 12,0 a $20,5 \mathrm{~kg}$, subdivididos em quatro grupos:

Grupo I) cinco cães submetidos a anóxia cardíaca por 60 minutos, em condiçōes normotérmicas;

Grupo II) cinco cães submetidos a anóxia cardíaca por 60 minutos, em condiçōes hipotérmicas $\left(28^{\circ} \mathrm{C}\right)$;

Grupo III) cinco cães submetidos a anóxia cardíaca por 60 minutos, em codiçōes hipotérmicas $\left(28^{\circ} \mathrm{C}\right)$, tendo recebido duas infusōes de soluçāo cardioplégica (soro glicosado como substrato básico);

Grupo IV) cinco cães submetidos a anóxia cardíaca por 60 minutos, em condiçōes hipotérmicas $\left(28^{\circ} \mathrm{C}\right)$, tendo recebido duas infusōes de solução cardioplégica (sangue como substrato básico).

\section{Técnica Cirúrgica}

Todos os animais foram submetidos a anestesia geral (tiopental sódico $33 \mathrm{mg} / \mathrm{kg}$ ), entubaçāo orotraqueal e mantidos com respiração controlada através de respirador Takaoka a volume, modelo 855 . A veia femoral direíta foi dissecada e cateterizada, possibilitando a infusão de líqüidos e drogas sempre que necessário, além de ser determinada a pressão venosa central. A artéria femoral direita foi igualmente dissecada e cateterizada, coneccionando-se a ela manômetro de mercúrio, o que permitia obterse continuamente pressão arterial média e possibilitava a colheita periódica de sangue para análises.

O coração e os vasos da base foram expostos através de toracotomia mediana transesternal. Após heparinização (3 mg/kg), estabeleceu-se circulação extracorpórea através da drenagem venosa de ambas as veias cavas, com introdução de cânulas de $1 / 4$ de polegada em meio a duas suturas em bolsa previamente realizadas no átrio direito, com fios de mononylon 4.0. As cavas superior e inferior foram dissecadas e cadarçadas, de maneira a desviar todo o retorno venoso para as cânulas posicionadas em seu interior. paralelamente, dissecou-se a artéria femoral esquerda e, através de sua canulação, foi processada a reinfusāo do sangue já oxigenado. Uma vez instalada a circulação extracorpórea, o átrio esquerdo foi drenado através de incisão em aurícula esquerda, evitando-se a distenção cardíaca. Empregaram-se bomba arterial de roletes e oxigenador de bolhas de coluna variável. A perfusāo foi iniciada com hemodiluição total através de solução de Ringer simples $(400 \mathrm{ml})$, dextran $(250 \mathrm{ml})$ e manitol $20 \%(50 \mathrm{ml})$, mantendo-se fluxo variável de 40 a $100 \mathrm{ml} / \mathrm{kg} / \mathrm{min}$.

Dosagens de hematócrito e hemoglobina, sódio e potássio e gasometria arterial foram realizadas previamente à toracotomia, a cada 30 minutos após sua realizaçāo, e sempre que alguma alteraçāo nos diversos parâmetros assim o justificasse. As correçōes foram processadas pela administração de bicarbonato de sódio a $10 \%$, cloreto de potássio $19,1 \%$, infusão de sangue homólogo ou através da variação no fluxo de oxigênio administrado.

\section{Solução Cardioplégica}

As soluçōes cardioplégicas empregadas foram baseadas na proposta por GOMES et alii ${ }^{7}$, a saber:

\section{Solução I:}

$\begin{array}{ll}\text { Soro glicosado } 5 \% & 1.000 \mathrm{ml} \\ \mathrm{NaCl} 30 \% & 68 \mathrm{mEq} / \mathrm{l} \\ \mathrm{KCL} 19,1 \% & 25 \mathrm{mEq} / \mathrm{l} \\ \mathrm{MgSO}_{4} 10 \% & 32 \mathrm{mEq} / \mathrm{l} \\ \mathrm{CaCl}_{2} 10 \% & 2,5 \mathrm{mEq} / \mathrm{l} \\ \text { Lidocaína }^{10} & 40 \mathrm{mg} / \mathrm{l} \\ \text { Verapamil } & 0,2 \mathrm{mg} / \mathrm{l} \\ \mathrm{NaHCO}_{3} 10 \% & 2,4 \mathrm{mEq} / \mathrm{l} \text { (q.s.p. } \mathrm{pH} \\ & \text { entre } 7,45 \text { e } 7,77 \text { ) } \\ \text { Osmolaridade } & 400-410 \mathrm{mOsm}\end{array}$

\section{Soluçăo II:}

Composição semelhante à solução I, sem o componentes $\mathrm{MgSO}_{4}$

\section{Soluçāo III:}

Composta pelos mesmos elementos da solução I, com exceção do veículo de infusão, constituído por sangue (do próprio cão ou de outro cão com reação cruzada compatível), ao invés de soro glicosado.

\section{Soluçāo IV:}

Semelhante à solução III, porém sem o componente $\mathrm{MgSO}_{4}$ 
DALLAN, L. A.; OLIVEIRA, S. A.; IGLÉZIAS, J. C. R.; PÊGO-FERNANDES, P. M.; AULER JÚNIOR, J. O. C.; VERGINELLI, G.; JATENE, A. D. - Soluçảo cardioplégica sangưínea versus acelular: avaliaçāo hemodinâmica através de estudo experimental em cảes. Rev. Bras. Cir. Cardiovasc., 5(3): 168-178, 1990.

A soluçăo cardioplégica foi administrada por punção da raíz da aorta após seu pinçamento, tendo sido utilizado volume de $15 \mathrm{ml} / \mathrm{kg} /$ peso e pressão de infusão em torno de $50 \mathrm{mmHg}$ na raíz da aorta.

As infusōes foram realizadas em duas etapas: a primeira imediatamente após o pinçamento da aorta e a segunda, decorridos 30 minutos dessa infusăo.

Essas soluçōes foram resfriadas entre $4^{\circ}$ e $10^{\circ} \mathrm{C}$, e a temperatura miocárdica, determinada por sensor térmico (Tele - thermometer Yellow Springs Instrument Co. Inc.) posicionado no septo interventricular. A temperatura miocárdica média dos cães dos Grupos III e IV variou de 9 a $14^{\circ} \mathrm{C}$, com média de $11,5^{\circ} \mathrm{C}$, enquanto que, nos Grupos I e II, a temperatura miocárdica média foi, respectivamente, $36,2^{\circ} \mathrm{C}$ e $29,1^{\circ} \mathrm{C}$.

\section{Estudo Hemodinâmico}

Sensores especiais para aferição de força contrátil do coração (strain - gauge, Arch - 120 OHM), acoplados a "transdutor" Hewlett Packard modelo 1280 - C, foram instalados epicárdio do ventrículo esquerdo (VE).

A aorta ascendente foi dissecada, tendo-se instalado em seu fluxômetro tipo garra, com diâmetro interno variável de 14 a $18 \mathrm{~mm}$ (Flo - Probe - Blood flow transducer Statham), acoplado a Blood Flowmeter modelo SP 2201 Statham), capaz de fornecer o fluxo sangüíneo obtido em cada sístole ventricular.

Cateteres tipo Lehman foram introduzidos no ventrículo esquerdo, através de sua ponta e acoplados via transdutores a polígrafo Hewlett Packard modelo $8890 \mathrm{~A}$, permitindo registrar as curvas pressóricas sisto - diastólicas de VE, juntamente com o eletrocardiograma (ECG) (Gráficos 1 a 4).

Cateteres semelhantes foram posicionados em tronco pulmonar através da via de saída de ventrículo direito. Into possibilitou a determinação do débito cardíaco pelo método dilucional (cárdio - green), através da infusão de $2,5 \mathrm{mg}$ da solução em tronco pulmonar e seu registro em aorta, utilizando-se analisador modelo Harvard Apparatus 901, seguido de leitura em densitômetro modelo Cardiac Output Analyser (Waters Instruments Inc.).

\section{GRÁFICO 1}

ECG E REGISTRO DE PRESSÓES SISTO-DIASTÓLICAS DE VE NAS CONDIÇŌES PRÉ-CEC (1), 30 (2) E 120 MINUTOS (3) PÓS-REPERFUSÃO MIOCÁRDICA. CĀO No 3 GRUPO I

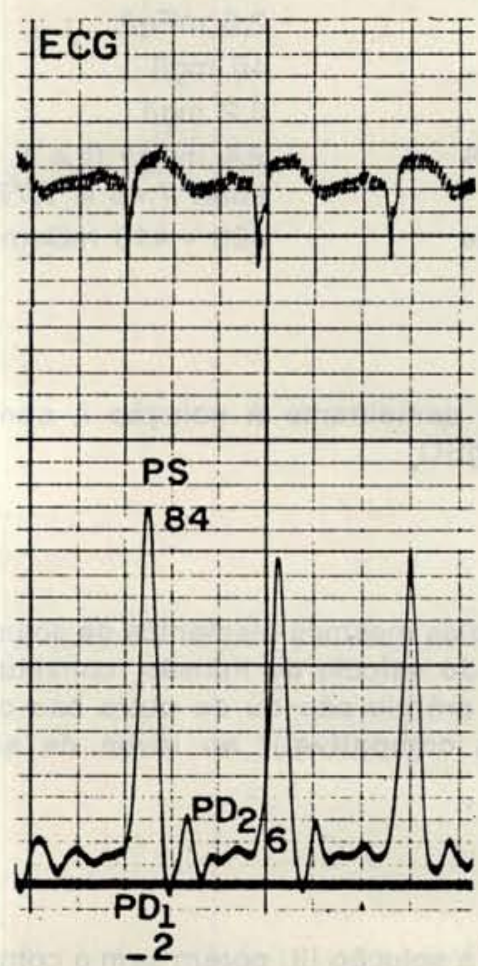

condição 1

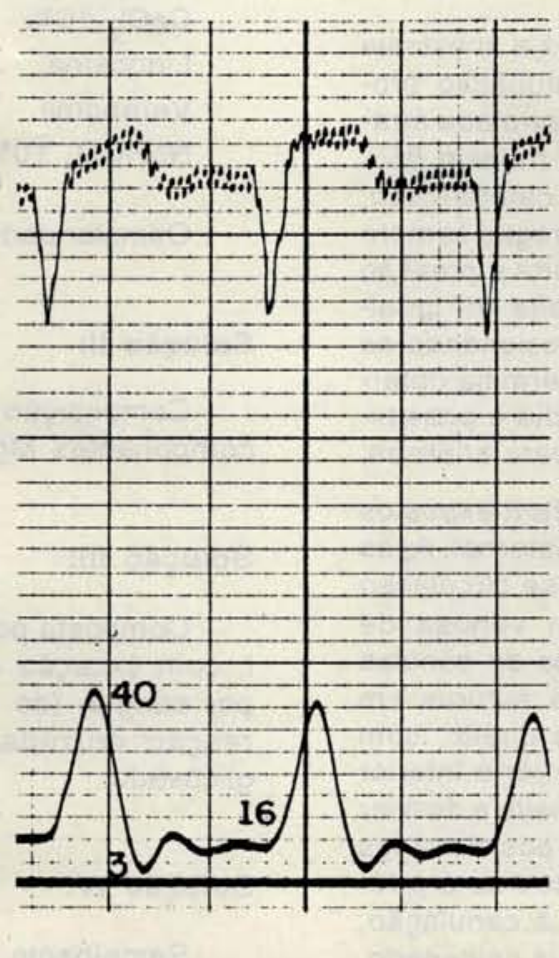

condição 2

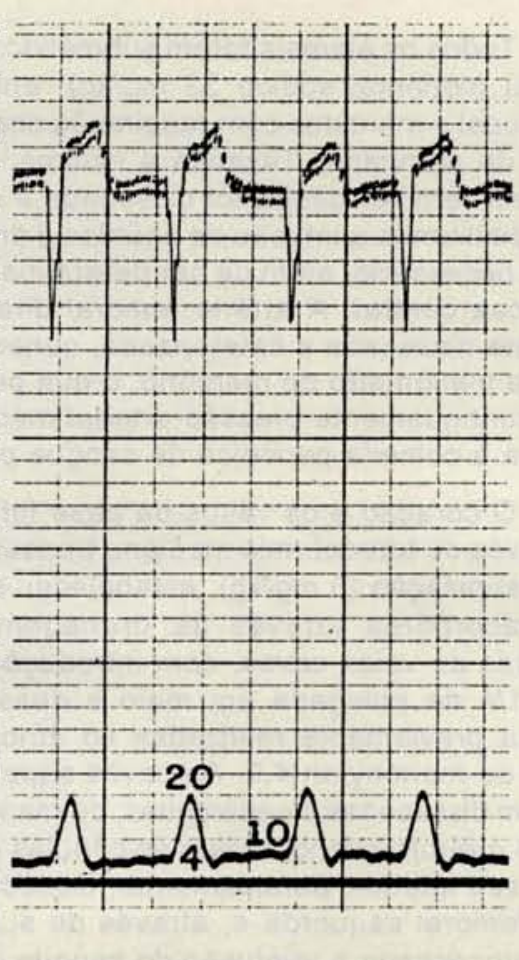

condição 3 
DALLAN, L. A.; OLIVEIRA, S. A.; IGLÉZIAS, J. C. R.; PÊGO-FERNANDES, P. M.; AULER JÚNIOR, J. O. C.; VERGINELLI, G.; JATENE, A. D. - Solução cardioplégica sangüínea versus acelular: avaliaçảo hemodinâmica através de estudo experimental em cảes. Rev. Bras. Cir. Cardiovasc., 5(3): 168-178, 1990.

\section{GRÁFICO 2}

ECG E REGISTRO DE PRESSŌES SISTO-DIASTÓLICAS DE VE NAS CONDIÇŌES PRÉ-CEC (1), 30 (2) E 120 MINUTOS (3) PÓS-REPERFUSĀO MIOCÁRDICA. CĀO N 4 GRUPO ॥

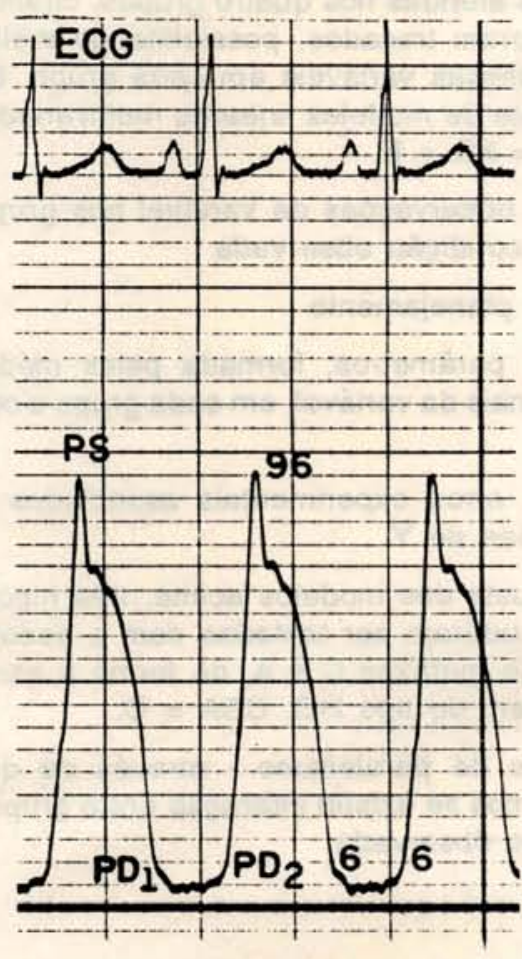

condição 1

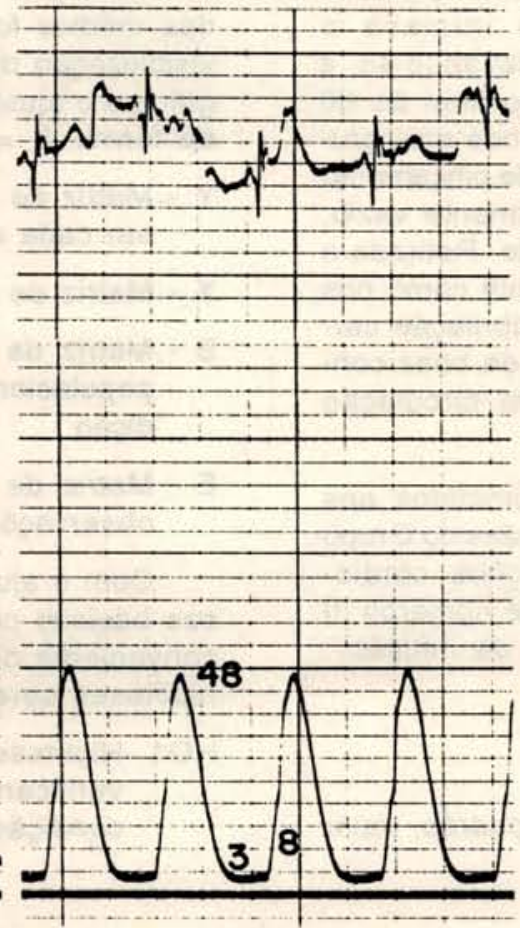

condição 2

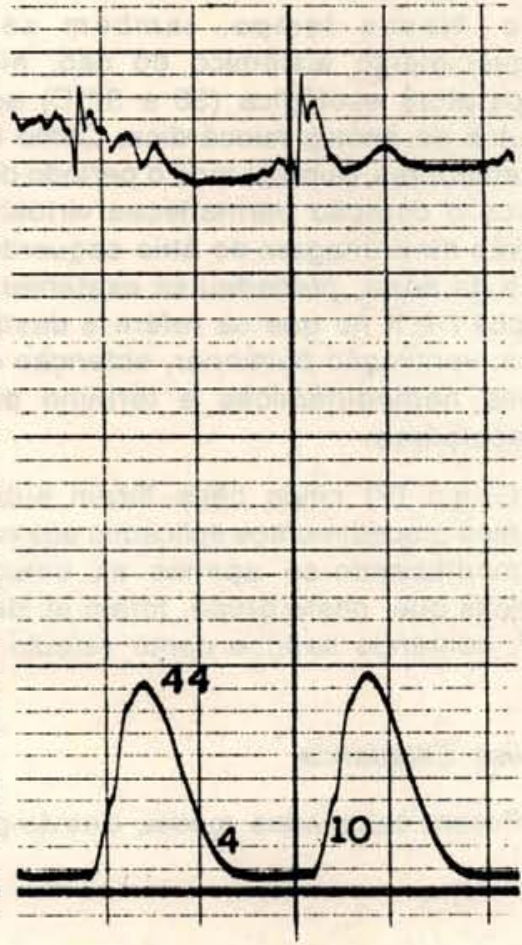

condição 3

\section{Procedimento nos Grupos}

Grupo I) cinco cães submetidos a circulação extracorpórea (CEC) e anóxia miocárdica normotérmica, através do pinçamento da aorta ascendente (acima dos óstios coronários) por período de 60 minutos. Decorrido este prazo, a aorta foi despinçada, com conseqüente reperfusāo do miocárdio. Os batimentos cardíacos restabeleceram-se espontaneamente ou através de desfibrilaçāo miocárdica direta. Normalizadas as condiçōes hemodinâmicas, os pulmōes foram ventilados, a aurícula esquerda suturada e a circulaçāo extracorpórea foi interrompida. Nos cinco cães desse grupo, após instalaçāo de eletrodos, sensores, fluxômetros e cateteres, como já descrito anteriormente, foram realizados os registros: ECG, força contrátil miocárdica, fluxo sangüíneo em aorta, curvas pressóricas sistodiastólicas em VE e débito cardíaco nos seguintes intervalos: pré CEC; 30 minutos pós reperfusāo miocárdica; 120 minutos pós reperfusāo miocárdica. Os valores foram obtidos no mínimo de três curvas, sendo utilizada sua média.

Grupo II) cinco cães submetidos a anóxia miocárdica hipotérmica $\left(28^{\circ} \mathrm{C}\right)$. Após instalada a circulaçāo extracorpórea, os animais foram esfria- dos progressivamente até $28^{\circ} \mathrm{C}$ através do permutador de calor do oxigenador, ocasiāo em que a aorta era pinçada por 60 minutos. O reaquecimento foi iniciado já aos 30 minutos de anóxia miocárdica, permitindo obter-se temperatura esofágica superior a $35^{\circ} \mathrm{C}$ ao final dos 60 minutos, quando se retirava a pinça da aorta. Os batimentos cardíacos foram obtidos espontaneamente ou através de desfibrilaçāo com pás internas. De maneira análoga ao Grupo I, aguardava-se bom restabelecimento da contratilidade cardíaca para a interrupção da CEC, mantendo-se os cães anestesiados e em ventilaçāo pulmonar assistida. Nos cinco cães foram realizados registros eletrocardiográficos, pressóricos, de força contrátil e débito cardíaco, antes da circulaçāo extracorpórea, 30 e 120 minutos após reperfusāo miocárdica.

Grupo III) cinco cães foram esfriados à temperatura de $28^{\circ} \mathrm{C}$ e submetidos a anóxia miocárdica por 60 minutos, aos quais se associou infusāo de solução cardioplégica entre 4 e $10^{\circ} \mathrm{C}$ na raíz da aorta, em duas etapas, num total de $15 \mathrm{ml} / \mathrm{kg}$ de peso. A primeira infusāo de soluçāo cardioplégica iniciava-se imediatamente após o pinçamento da aorta, utilizando-se a soluçāo cardioplégica I. A pressāo de infusão era mantida em torno de $50 \mathrm{mmHg}$ na raíz da aorta, com fluxo inicial mais intenso, 
DALLAN, L. A.; OLIVEIRA, S. A.; IGLÉZIAS, J. C. R.; PÊGO-FERNANDES, P. M.; AULER JÚNIOR, J. O. C.; VERGINELLI, G.; JATENE, A. D. - Soluçāo cardioplégica sangüínea versus acelular: avaliação hemodinâmica através de estudo experimental em cảes. Rev. Bras. Cir. Cardiovasc., 5(3): 168-178, 1990.

visando fechar a valva aórtica e propiciar a imediata parada cardíaca em diástole. Decorridos 30 minutos, o procedimento era repetido, com a soluçāo cardioplégica II, até totalizar-se o volume preconizado. Nesse tempo, também se iniciava o reaquecimento sistêmico do cão, elevando-se a temperatura esofágica $\left(35\right.$ a $\left.37^{\circ} \mathrm{C}\right)$ ao final de 60 minutos de anóxia miocárdica. Como nos dois grupos anteriores, durante todo o período de pinçamento aórtico o coraçāo permaneceu virtualmente vazio, através da drenagem do átrio esquerdo. Retirada a pinça da aorta, procedeu-se exatamente como nos Grupos I e II no que se refere a desfibrilação cardíaca, ventilação pulmonar, obtenção de boas condiçōes hemodinâmicas e término da circulaçāo extracorpórea.

Grupo IV) cinco cães foram submetidos aos mesmos procedimentos aplicados aos cães do Grupo III, modificando-se apenas as soluçōes cardioplégicas que, neste grupo, foram as de números III e IV, contendo sangue como veículo de infusão.

\section{Análise Estatística}

Foram calculados média, desvio-padrāo, valor mínimo e máximo das variáveis $\mathrm{CK}, \mathrm{CKMB}$, pressão diastólica inicial $\left(P D_{1}\right)$, pressāo diastólica final $\left(\mathrm{PD}_{2}\right)$, pressão (PS), débito cardíaco (DC), em cada uma das condiçōes aferidas nos quatro grupos. Gráficos das médias foram traçados, possibilitando melhor visibilizaçāo dessas variáveis em cada grupo. Seguiu-se 0 ajuste de modelos lineares multivariados, da forma: $Y=X B+E$.

Y - Matriz de observaçōes da variável nos grupos em cada condiçāo observada

$X$ - Matriz de planejamento

B - Matriz de parâmetros, formada pelas médias populacionais da variável, em cada grupo e condição

E - Matriz de erros experimentais associados às observaçōes de $Y$

Com o ajuste dos modelos acima, três hipóteses básicas puderam ser testadas com a escolha conveniente de matrizes $\mathrm{C}$ e $\mathrm{A}$, de forma a essas hipóteses serem do tipo HO: CBA $=0$.

HO1 - Hipótese de paralelismo - através da qual verificamos se existiu interaçāo entre grupo e condição observada.

\section{GRÁFICO 3}

ECG E REGISTRO DE PRESSŌES SISTO-DIASTÓLICAS DE VE NAS CONDIÇŌES PRÉ-CEC (1), 30 (2) E 120 MINUTOS (3) PÓS-REPERFUSĀO MIOCÁRDICA. CĀO NN 1 GRUPO III

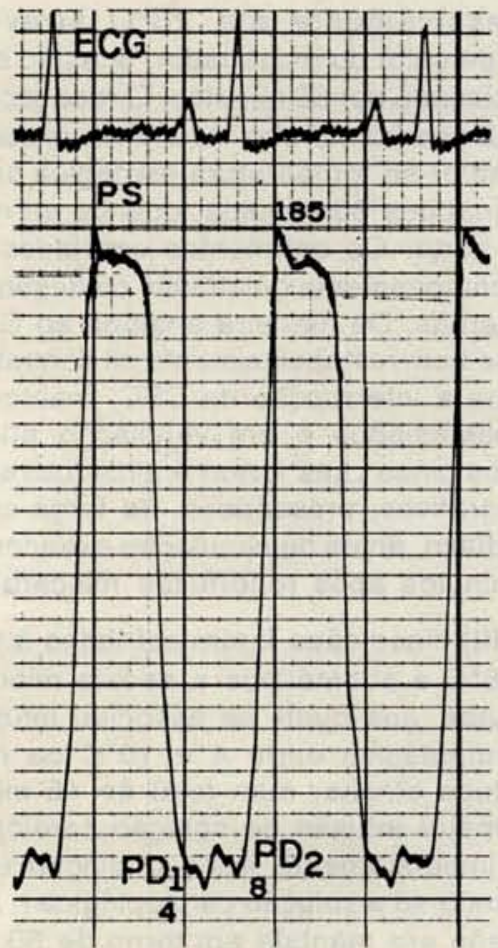

condição 1

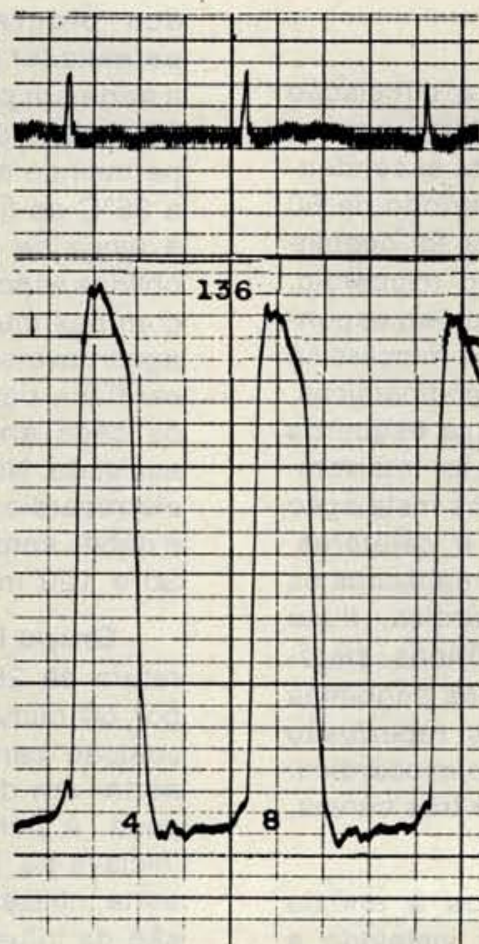

condição 2

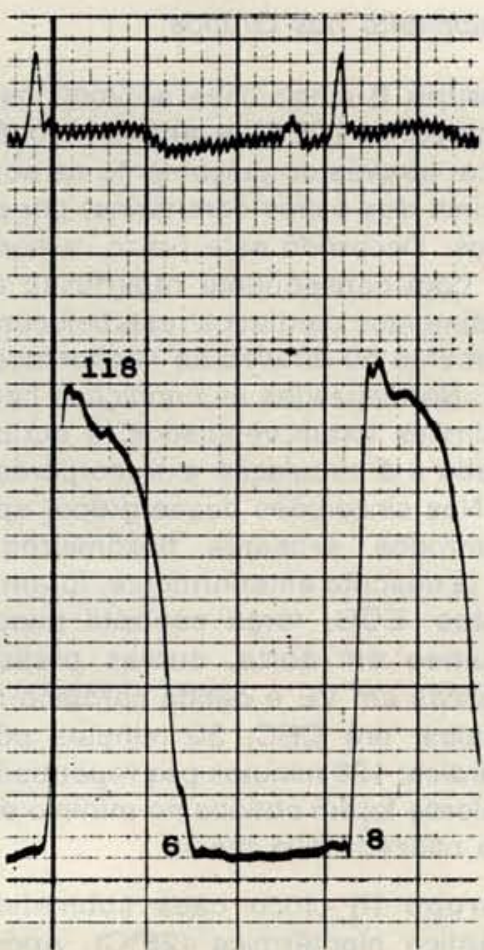

condição 3 
DALLAN, L. A.; OLIVEIRA, S. A.; IGLÉZIAS, J. C. R.; PÊGO-FERNANDES, P. M.; AULER JÚNIOR, J. O. C.; VERGINELLI, G.; JATENE, A. D. - Soluçāo cardioplégica sangūínea versus acelular: avaliaçāo hemodinâmica através de estudo experimental em cães. Rev. Bras. Cir. Cardiovasc., 5(3): 168-178, 1990.

\section{ECG E REGISTRO DE PRESSŌES SISTO-DIASTÓLICAS DE VE NAS CONDIÇŌES PRÉ-CEC (1), 30 (2) E 120 MINUTOS (3) PÓS-REPERFUSĀO MIOCÁRDICA. CĀO N² GRUPO IV}

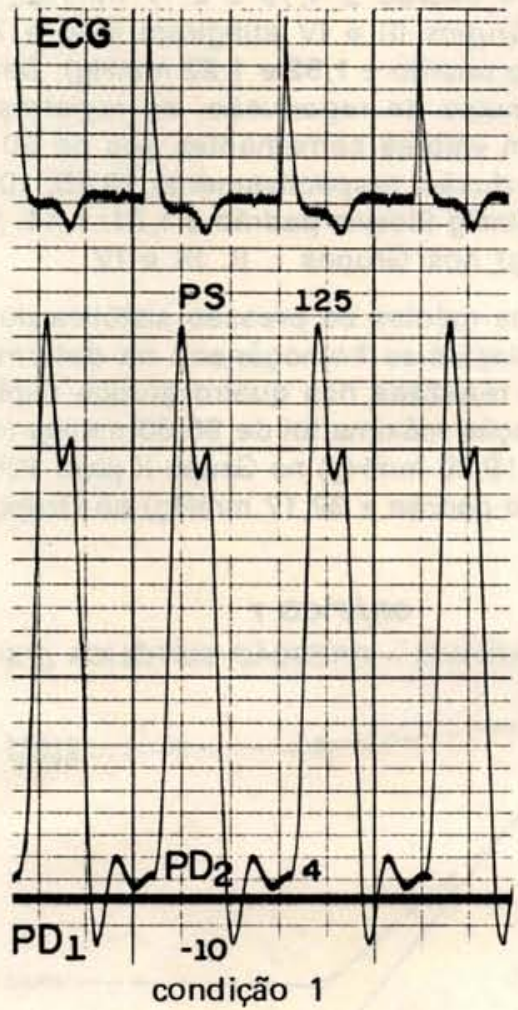

condição 1

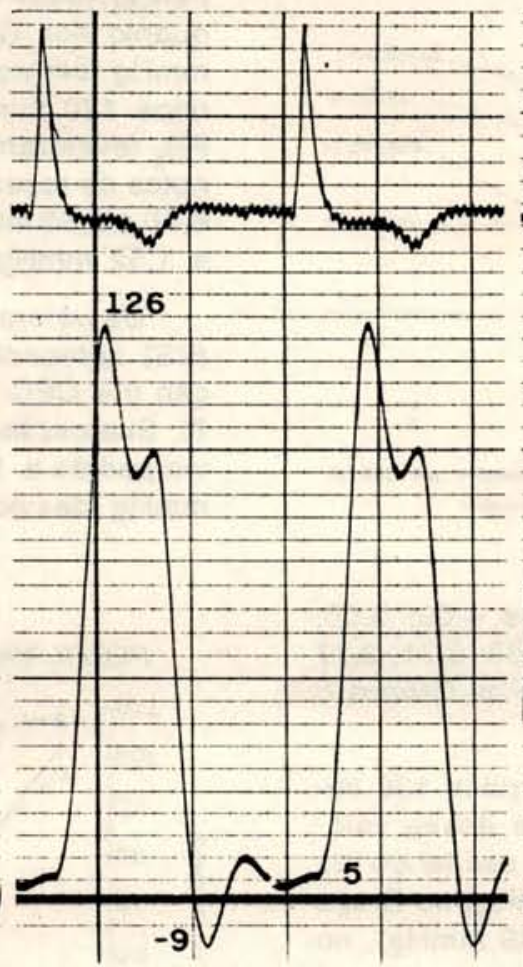

condição 2

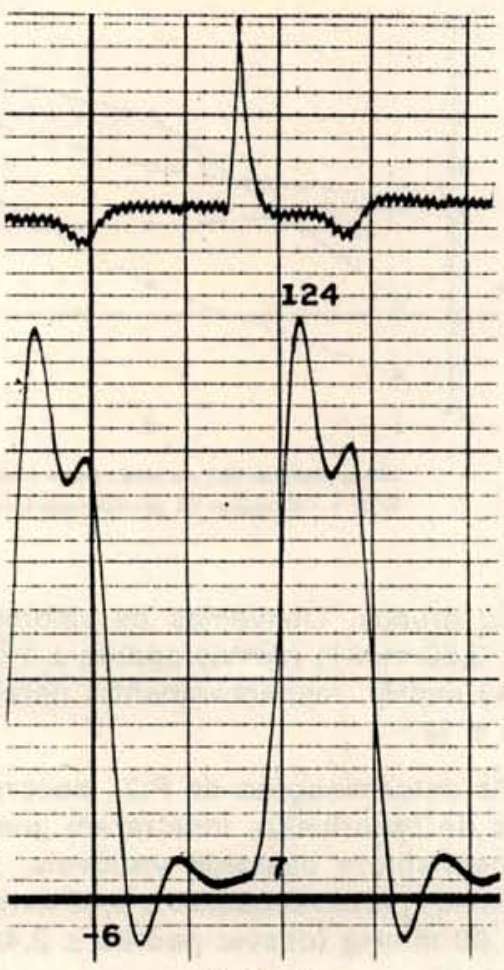

condição 3
HO2 -Hipótese de igualdade de grupos - através da qual verificamos se o comportamento dos grupos doi idêntico.

HO3 -Hipótese de igualdade de condiçōes - através da qual verificamos se comportamento da variável em estudo não diferiu nas condiçōes em que foi observada.

Ao conjunto dessas três hipóteses, denominamos análise de perfil (TIMM ${ }^{17}$ ).

Nos casos em que a hipótese $\mathrm{HO} 2$ foi estatisticamente significativa, prosseguimos na análise dos grupos, na busca da identificaçăo de quais diferiam entre si. Foram comparados os quatro grupos, dois a dois.

Quando observamos diferença entre condiçōes pela hipótese $\mathrm{HO} 3$, também houve necessidade de se prosseguir a análise, comparando-se as várias condiçōes de acordo com o interesse. Tais comparaçōes foram efetuadas por grupo ou por conjunto de grupos, de acordo com os resultados já obtidos.

A suposiçāo necessária para os ajustes propostos foi de que os erros experimentais tiveram distribuiçāo normal multivariada, com vetor de média nulo e matriz de covariança desconhecida. Esta suposiçāo foi verificada através da análise de resíduos.

Todos os cálculos feitos utilizando-se o procedimento REG do software estatístico S.A.S. (Statistical Analysis System, 1982) ${ }^{16}$.

\section{RESULTADOS}

Os valores descritivos médios, desvio-padrão, mínimo e máximo correspondestes às variáveis $P D_{1}$, $P D_{2}, P S$ e $D C$, por grupo e condição, estão representados nos Gráficos 5, 6, 7 e 8 . Esses dados foram obtidos através do registro de pressōes sistodiastólicas do VE (Gráficos 1 a 4) e da determinaçāo do débito cardíaco.

Pudemos observar que o valor médio de $P D_{1}$ apresentou grande variaçāo nas três condiçōes em que foi determinado (Gráfico 5). Antes de instalada a CEC, obtivemos valores que oscilaram de 2,40 $\mathrm{mmHg}$ (desvio padrão $\pm 4,83 \mathrm{mmHg}$ ), determinado no Grupo II a $-1,20 \mathrm{mmHg}$ (desvio padrão $\pm 5,36$ $\mathrm{mmHg}$ ), determinando no Grupo IV. Os registros de $P D$, realizados 30 minutos após reperfusão miocárdica também não foram homogêneos nos 
DALLAN, L. A.; OliVEIRA, S. A.; IGLÉZIAS, J. C. R.; PÊGO-FERNANDES, P. M.; AULER JÚNIOR, J. O. C.; VERGINELLI, G.; JATENE, A. D. - Solução cardioplégica sangüínea versus acelular: avaliação hemodinâmica através de estudo experimental em cảes. Rev. Bras. Cir. Cardiovasc., 5(3): 168-178, 1990.

GRÁFICO 5

PERFIL VARIÁVEL - PRESSÃO DIASTÓLICA INICIAL (PD1)

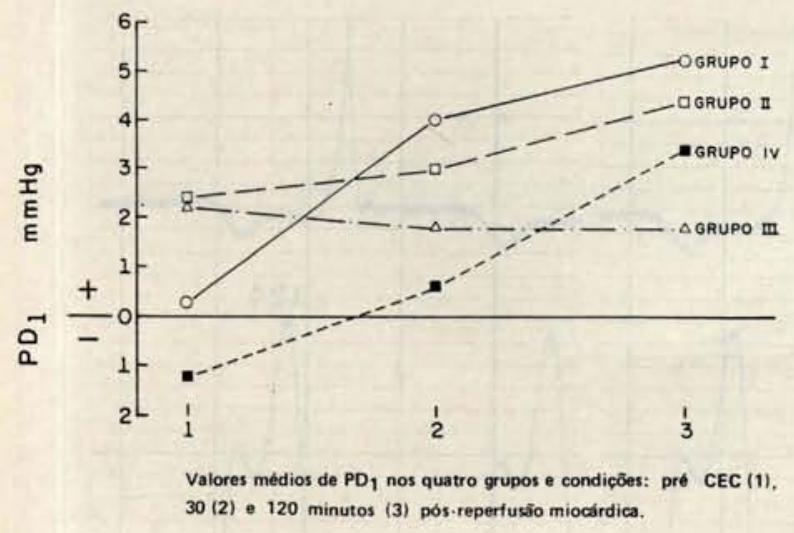

quatro grupos. Obtivemos os valores 4,$00 ; 3,00$; 1,$80 ; 0,60 \mathrm{mmHg}$ (desvio padrāo $\pm 1,83 ; 0,71 ; 3,27$ e $6,43 \mathrm{mmHg}$, respectivamente) para os Grupos I, II, III e IV.

As determinaçōes de $P D_{1}$, decorridos $120 \mathrm{mi}-$ nutos de reperfusão, mostraram que esses valores persistiram bastante variáveis, oscilando de $5,25 \mathrm{mmHg}$ (desvio padrāo $\pm 3,40 \mathrm{mmHg}$ ) no Grupo I a $1,80 \mathrm{mmHg}$ (desvio padrāo $\pm 2,49 \mathrm{mmHg}$ ), no Grupo III.

Os valores médios de $\mathrm{PD}_{2}$ obtidos antes da instalaçāo da CEC (controles) foram homogêneos nos quatro grupos de cāes, oscilando entre 4,5 $\mathrm{mmHg}$ (desvio padrāo $\pm 3,11 \mathrm{mmHg}$ ) no grupo I e $6,00 \mathrm{mmHg}$ (desvio padrão $\pm 1,58 \mathrm{mmHg}$ ) no Grupo III, (Grágico 6). As determinaçōes de $P D_{2}$ ao final

\section{GRÁFICO 6}

PERFIL VARIÁVEL - PRESSĀO DIASTÓLICA FINAL (PD2)

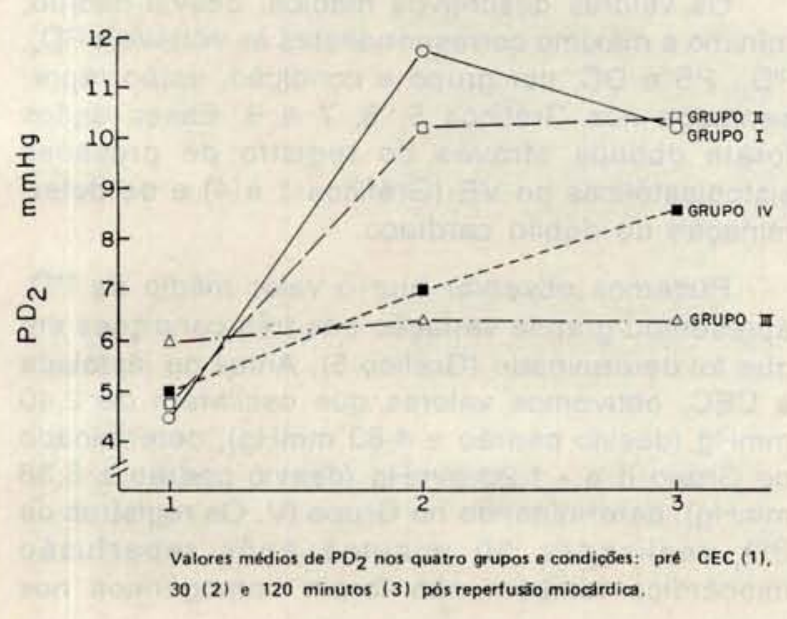

de 30 minutos de reperfusão, entretanto, evidenciaram sua elevação, especialmente nos Grupos I e II tendo atingido, respectivamente, 11,75 e 10,20 $\mathrm{mmHg}$ (desvio padrāo $\pm 4,19$ e $2,17 \mathrm{mmHg}$ ), enquanto nos Grupos III e IV atingiram 6,40 e 7,00 $\mathrm{mmHg}$ (desvio padrāo $\pm 1,52$ e 1,22 mmHg). Decorridos 120 minutos de reperfusāo, os registros de $\mathrm{PD}_{2}$ revelaram valores semelhantes aos de $30 \mathrm{mi}-$ nutos de reperfusāo, respectivamente 10,$25 ; 10,40$; 6,40 e $8,60 \mathrm{mmHg}$ (desvio padrāo $\pm 1,71 ; 1,14 ; 1,52$ e $1,52 \mathrm{mmHg}$ ) nos Grupos I, II, III e IV.

Os valores médios da pressāo sistólica do VE (PS) apresentaram-se homogêneos na determinação pré CEC realizada nos quatro grupos (Gráfico 7). Sua oscilação máxima foi de $96,80 \mathrm{mmHg}$ (desvio padrão $\pm 19,30 \mathrm{mmHg}$ ) no Grupo II para 108,20 $\mathrm{mmHg}$ (desvio padrāo $\pm 32,17 \mathrm{mmHg}$ ) no Grupo III.

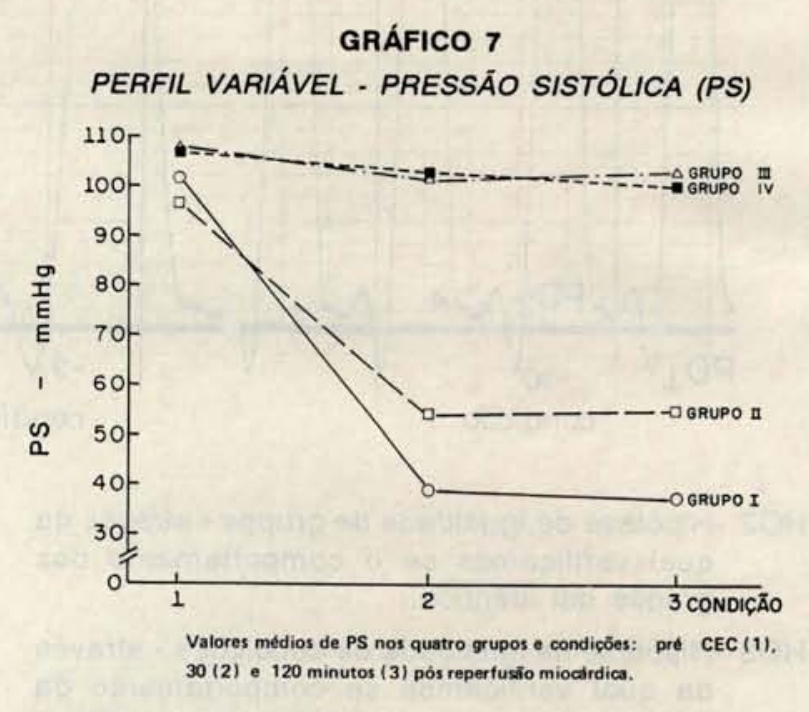

Decorridos 60 minutos de anóxia e 30 minutos de reperfusão miocárdica, entretanto, observamos queda acentuada da PS nos Grupos I e II, tendo atingido, respectivamente, 39,00 e $54,40 \mathrm{mmHg}$ (desvio padrảo $\pm 10,89$ e 11,35 $\mathrm{mmHg}$ ). Nos Grupos III e IV essa variaçāo foi mínima, com valores, respectivamente, de 102,40 e $103,60 \mathrm{mmHg}$, desvio padrāo $\pm 31,86$ e $30,06 \mathrm{mmHg}$. Os registros da PS realizados ao final de 120 minutos de reperfusão mostraram valores semelhantes, respectivamente, 32,$75 ; 55,00 ; 103,80 ; 100,80 \mathrm{mmHg}$ (desvio padrāo $\pm 15,39 ; 9,95 ; 14,89$ e $33,91 \mathrm{mmHg}$ ) nos Grupos I, II, III e IV.

A determinaçāo do débito cardíaco (DC) antes da instalação da CEC (controle) revelou valores bastante semelhantes nos quatro grupos de cães, com oscilaçāo máxima de 1,93 para $2,18 \mathrm{l} / \mathrm{min}$., (desvio padrāo $\pm 0,26$ e $0,36 \mathrm{l} / \mathrm{min}$.), respectivamente 
DALLAN, L. A.; OliVeIRA, S. A.; IGLÉZIAS, J. C. R.; PÊGo-FERNANDES, P. M.; AULER JÚNIOR, J. O. C.; VERGINELLI, G.; JATENE, A. D. - Solução cardioplégica sangūínea versus acelular: avaliaçāo hemodinâmica através de estudo experimental em cäes. Rev. Bras. Cir. Cardiovasc., 5(3): 168-178, 1990.

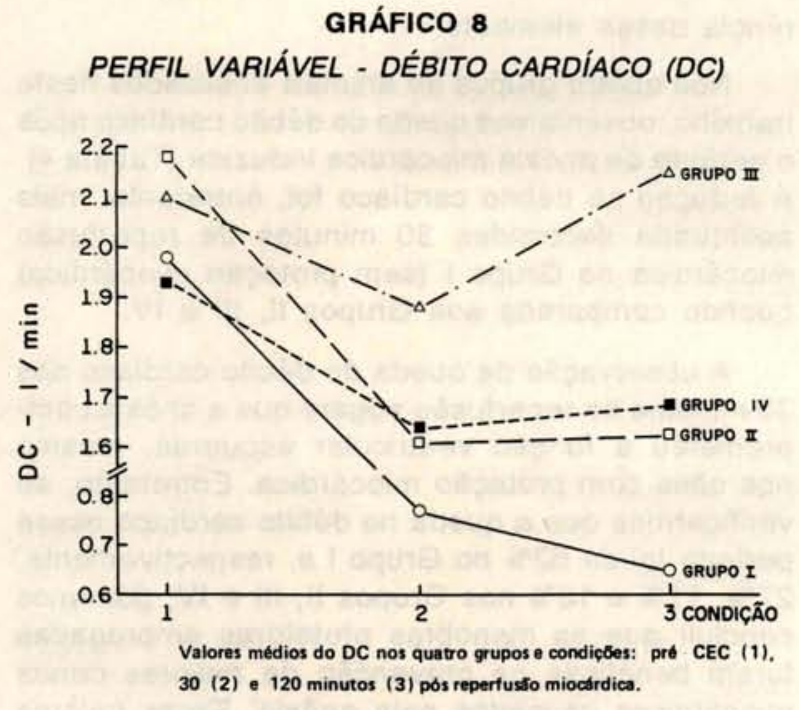

nos Grupos II e IV (Gráfico 8). Decorridos $30 \mathrm{mi}$ nutos de reperfusão, observamos queda do DC nos quatro grupos, com valores, respectivamente, de 0,$77 ; 1,61 ; 1,88$ e $1,64 \mathrm{l} / \mathrm{min}$. (desvio padrāo $\pm 0,55$; 0,$45 ; 0,13$ e $0,16 \mathrm{I} / \mathrm{min}$.) nos Grupos I, II, III e IV. A determinação do $\mathrm{DC}$ aos 120 minutos de reperfusão evidenciou queda ainda mais acentuada no Grupo I atingindo $0,65 \mathrm{I} / \mathrm{min}$. (desvio padrāo \pm $0,79 \mathrm{l} / \mathrm{min}$.). Nos demais grupos, entretanto, houve recuperaçāo em seu valor, respectivamente 1,63; 2,15 e $1,69 \mathrm{l} / \mathrm{min}$. (desvio padrão $\pm 0,47 ; 0,17$ e $0,27 \mathrm{I} / \mathrm{min}$.) nos Grupos II, III e IV.

Foram calculados os dados referentes às análises de perfil de cada variável e as respectivas descriçōes dos testes das hipóteses $\mathrm{HO}, \mathrm{HO} 2$ e HO3. A cada hipótese testada foram obtidos os valores da estatística de Wilks (W), da aproximação para a estatística de Fischer - Snedecor (F), além do nível de probabilidade a ela associada (p). Pudemos observar que as hipóteses $\mathrm{HO} 2, \mathrm{HO}$, que estudam o comportamento dos grupos e das variáveis hemodinâmicas nas diferentes condiçōes testadas, apresentaram alto grau de significação $(p<$ $0,05)$ nas medidas da $P D_{2}$, PS e DC.

Em relação à variável $P D_{1}$, entretanto, não observamos diferença em seu comportamento entre os quatro grupos (hipótese $\mathrm{HO} 2$ ) e nas três determinações realizadas (hipótese $\mathrm{HO}$ ), pois seu nível de probalidade não foi significativo ( $p$ respectivamente 0,4663 e 0,0613 ).

Foram realizadas comparaçōes (duas a duas) entre as variáveis $\mathrm{PD}_{2}, \mathrm{PS}$ e $\mathrm{DC}$ nos diversos grupos onde a hipótese $\mathrm{HO} 2$ foi significativa. Os valores da variável $\mathrm{PD}_{2}$ divergiram estatisticamente $(p<0,05)$ entre os Grupos I e III $(p=0,0015)$, I e IV ( $p=0,0293$ e II e III $(p=0,0022)$, sendo semelhantes nos demais. Os valores da variável PS somente foram semelhates estatisticamente ao compararmos os Grupos III e IV ( $p=0,9565)$, diferindo nos demais. A variável $D C$ apresentou valores comparáveis entre os Grupos II e III ( $p=$ 0,1874 , II e IV $(p=0,2815)$ e III e IV $(p=0,4636)$. O Grupo I, entretanto, apresentou sempre diferença significativa $(p<0,05)$ quando comparado aos demais.

Visando à simplificação da análise estatística, os quatro grupos foram reagrupados segundo a semelhança de cada variável. Dessa maneira, a variável $P D_{2}$ passou a ser analisada sob dois novos agrupamentos, sendo o primeiro (A) constituído pelos Grupos I e II, e o segundo (B) pelos Grupos III e IV. A variável PS foi também recomposta em dois novos agrupamentos, pois os Grupos I e II (C) e III e IV (D) apresentavam comportamentos semelhantes. Pudemos reagrupar a variável $\mathrm{DC}$ em dois novos conjuntos, compostos, respectivamente, pelo Grupo I (F) e pelos Grupos II, III e IV (G).

Foram calculados os resultados das comparaçōes de interesse realizadas para os novos agrupamentos com os respectivos valores estatísticos e níveis de probabilidade. A variável $\mathrm{PD}_{2}$ apresentou elevação significativa em sua média $(p<0,05)$ no reagrupamento $A$ (Grupos I e II) quando comparada às condiçōes pré CEC e 30 minutos $(p<0,0001)$ e pré CEC e 120 minutos de reperfusão miocárdica $(p<0,0001)$. Entre 30 e 120 minutos de reperfusão, entretanto, a elevação de $\mathrm{PD}_{2}$ não foi significativa $(p=0,3373$. No reagrupamento $B$ (Grupos III e IV) a elevação de $\mathrm{PD}_{2}$ foi significativa apenas na condição pré $C E C$ e 120 minutos de reperfusão $(p=$ $0,0023)$.

A variável PS apresentou queda significativa aos 30 minutos $(p<0,0001)$ e 120 minutos $(p<$ 0,0001 ) de reperfusão miocárdica no reagrupamento C (Grupos I e II). O mesmo não ocorreu no reagrupamento $D$ (Grupos III e IV), onde não observamos variação significativa $(p<0,05)$ entre as condiçōes analisadas.

A comparação de interesse do reagrupamento E (Grupo I) para a variável DC demonstrou sua queda significativa entre as condiçōes pré $C E C$ e 30 minutos $(p<0,0001)$ e pré CEC e 120 minutos de reperfusão miocárdica $(p<0,0001)$. Entre 30 e 120 minutos de reperfusão não houve variação significativa $(p=0,3409)$. No reagrupamento $F$ (Grupos II, III e IV) também observamos queda significativa do DC ao compararmos a condição pré CEC com 30 minutos $(p<0,0001)$ e com 120 minutos de reperfusão miocárdica $(p=0,0294)$, enquanto que entre 30 e 120 minutos de reperfusão não houve variaçāo significativa do $D C(p=0,1278)$.

O Gráfico 9 exprime a curva de funçāo ven- 
DALLAN, L. A.; OLIVEIRA, S. A.; IGLÉZIAS, J. C. R.; PÊGO-FERNANDES, P. M.; AULER JÚNIOR, J. O. C.; VERGINELLI, G.; JATENE, A. D. - Soluçāo cardioplégica sangüínea versus acelular: avaliaçāo hemodinâmica através de estudo experimental em cảes. Rev. Bras. Cir. Cardiovasc., 5(3): 168-178, 1990.

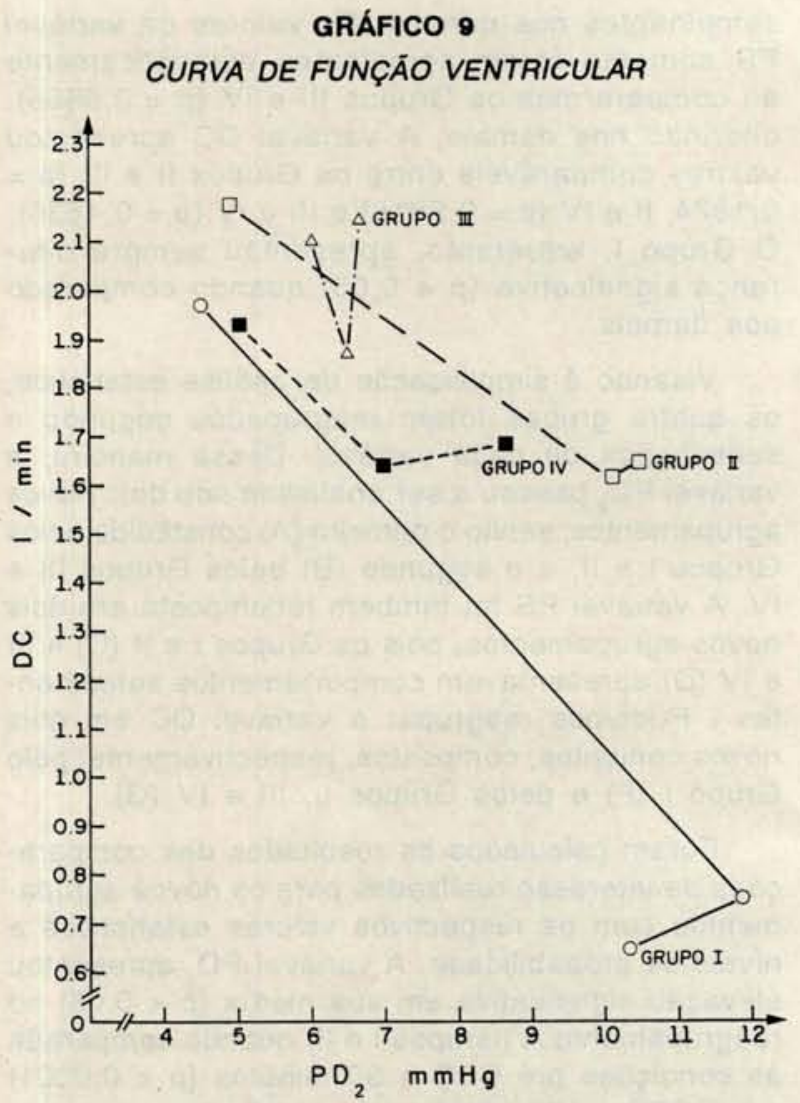

Curva de Função Ventricular dos animais dos quatro grupos nas três condiçōes estudadas (pré CEC, 30 e 120 minutos pós reperfusão miocárdica), integrando os valores médios do DC e da PD2 do VE.

tricular dos animais dos quatro grupos nas três condiçōes estudadas (pré CEC, 30 minutos e 120 minutos de reperfusāo miocárdica), integrando os valores do $\mathrm{DC}$ e da $P D_{2}$ do VE.

\section{COMENTÁRIOS}

O débito cardíaco constitui-se em parâmetro adequado para a avaliaçāo da contratilidade miocárdica (YANG et alii ${ }^{18}$ ). Embora seja funçāo direta do estado contrátil do VE, existem outros fatores que podem influir em seu valor final, especialmente a freqüência cardíaca, a distençāo a que a fibra cardíaca está submetida (pré-carga) e a resistência à ejeçāo do VE (pós-carga) (KARLINER et alii ${ }^{10}$ ). Para diminuir a influência destas variáveis sobre o débito cardíaco, procuramos manter todos os animais dentro de parâmetros próximos do normal, no que diz respeito à volemia, ao conteúdo hemoglobínico e sua saturaçāo, à pressāo arterial e às condiçōes metabólicas. Por outro lado, a análise comparativa dos pesos dos cāes mostrou semeIhança nos quatro grupos, o que nos permitiu interpretar os valores do débito cardíaco sem a interfe- rência desse elemento.

Nos quatro grupos de animais analisados neste trabalho, observamos queda do débito cardíaco após o período de anóxia miocárdica induzida (Tabela 4). A reduçāo no débito cardíaco foi, entretanto, mais acentuada decorridos 30 minutos de reperfusāo miocárdica no Grupo I (sem proteçāo miocárdica) quando comparada aos Grupos II, III e IV.

A observação da queda do débito cardíaco aos 30 minutos de reperfusăo sugere que a anóxia comprometeu a função ventricular esquerda, mesmo nos cães com proteção miocárdica. Entretanto, ao verificarmos que a queda no débito cardíaco nesse período foi de $62 \%$ no Grupo I e, respectivamente, $27 \%, 11 \%$ e $15 \%$ nos Grupos II, III e IV, podemos concluir que as manobras protetoras empregadas foram benéficas na prevençāo de maiores danos miocárdicos causados pela anóxia. Esses valores assumem expressāo ainda maior se lembrarmos que dois dos cāes do Grupo I nāo conseguiram manter-se sem o auxílio da circulaçāo extracorpórea após a anóxia, nāo tendo sido computados quando da análise estatística que determinou a média dos débitos.

Decorridos 120 minutos de reperfusāo, o débito cardíaco teve tendência à estabilizaçāo nos quatro grupos, embora em niveis mais baixos que os iniciais (Gráfico 8). Nos Grupos II, III e IV houve discreta elevaçāo do débito cardíaco em relação ao determinado com 30 minutos de reperfusāo. Embora sem significado estatístico, interpretamos esse fato como recuperaçāo da funçāo mecânica do VE, provalvemente por seu menor comprometimento funcional. No Grupo I, entretanto, observamos, nesse período, queda adicional do débito cardíaco.

A pressão diastólica final do VE $\left(\mathrm{PD}_{2}\right)$ não variou paralelamente ao débito cardíaco. O comportamento da $\mathrm{PD}_{2}$ nos Grupos I e II mostrou-se semelhante, diferindo nos Grupos III e IV, que, por sua vez, foram iguais. Nos primeiros houve aumento significativo da média de $\mathrm{PD}_{2}$, quando comparada às condiçōes pré CEC e 30 minutos, pré CEC e 120 minutos. Considerando que procuramos minimizar as variaçōes da pré e da pós carga através da padronizaçāo pressórica e volêmica nos quatro grupos, devemos atribuir, preferencialmente, ao déficit contrátil do VE tal elevaçāo (GROSSMAN et alii ${ }^{8}$ ). Nāo observamos diferença de $\mathrm{PD}_{2}$ nos tempos 30 e 120 minutos de reperfusāo nos Grupos I e II, possivelmente pelo fato da mesma já se encontrar bastante elevada, sem que houvesse sinal de recuperação do VE.

Nos Grupos III e IV nāo houve elevaçăo de $\mathrm{PD}_{2}$ aos 30 minutos, mas sim aos 120 minutos. Acreditamos que, nāo havendo lesāo miocárdica impor- 
DALLAN, L. A.; OlIVEIRA, S. A.; IGLÉZIAS, J. C. R.; PÊGO-FERNANDES, P. M.; AULER JÚNIOR, J. O. C.; VERGINELLI, G.; JATENE, A. D. - Soluçāo cardioplégica sangūínea versus acelular: avaliaçāo hemodinâmica através de estudo experimental em cães. Rev. Bras. Cir. Cardiovasc., 5(3): 168-178, 1990.

tante nesses grupos, os coraçōes desses cāes continuaram a comportar-se como "normais" e, portanto, com maior possibilidade de ativarem seus mecanismos de adaptação ventricular à nova condiçāo hemodinâmica, sem elevaçăo significativa inicial de $\mathrm{PD}_{2}$ (BRAUNWALD ${ }^{2}$ ). Năo interpretamos a elevaçāo de $\mathrm{PD}_{2}$ decorridos 120 minutos de reperfusāo como deterioraçāo da funçāo ventricular e sim pelos mesmos mescanismos de adaptaçāo. Como esse período coincidiu com a total reposiçāo volêmica nos cães, esse fato, associado à diminuiçăo da freqüência cardíaca, pode ter contribuído para tal elevação. Vem de encontro a essa hipótese o fato da pressāo sistólica ventricular esquerda (PS) não ter apresentado qualquer variação significativa em nenhuma das condiçōes observadas nos cães dos Grupos III e IV, denotando que, embora haja elevaçāo da $P D_{2}$, ainda existe reserva miocárdica para mantê-la estável.

O mesmo não ocorreu com os animais dos Grupos I e II. Houve queda significativa da PS aos 30 e 120 minutos de reperfusāo, quando comparadas à fase pré-toracotomia, possivelmente pela lesão miocárdica anóxica. Além disso, três dos cinco cães estudados no Grupo I receberam drogas inotrópicas, o que proporcionou PS artificialmente mais elevada. Não houve diferença estatística de PS nos tempos 30 e 120 minutos de reperfusão nesses dois primeiros grupos, exatamente por já se encontrar excessivamente baixa.

Não nos foi possível analisar com maior detaIhes os valores de $P D_{1}$, devido à grande variabili- dade nos dados obtidos, tanto nos quatro grupos, como nas três condiçōes estudadas.

Procuramos sintetizar as informaçōes hemodinâmicas obtidas, transportando-as para uma curva de funçăo ventricular, cuja expressão poderá ser observada no Gráfico 9 , que integra as tabelas de débito cardíaco e pressão diastólica final de VE $\left(P D_{2}\right)$. Nele verificamos uma nítida separação entre - Grupo I e os demais. Podemos observar em todos os grupos uma depressão da curva de função ventricular 30 minutos após a anóxia induzida, que é, entretanto, bastante mais acentuda no Grupo I. Decorridos 120 minutos de reperfusão miocárdica notamos tendência à melhora desta função nos Grupos II, III e IV, enquanto que, no grupo sem proteçāo miocárdica a depressāo inicial tende a agravar-se, afastando ainda mais sua curva de funçāo dos demais grupos.

Concluímos que os animais submetidos a isquemia miocárdica em normotermia (Grupo I) apresentaram intenso comprometimento na função do miocárdio. A hiportemia sistêmica isolada $\left(28^{\circ} \mathrm{C}\right)$, precedendo à indução da isquemia miocárdica (Grupo II), nāo foi suficiente para propiciar boa proteçāo ao miocárdio. Por outro lado, os dois tipos de solução cardioplégica empregados e associados à hipotermia (Grupos III e IV) preservaram eficazmente a funçāo ventricular. Entretanto, para o período de anóxia miocárdica empregada não obtivemos valores estatísticos significativos que possibilitassem inferir a superioridade da solução cardioplégica sangüínea sobre a acelular.

RBCCV 44205-121

DALLAN, L. A.; OLIVEIRA, S. A.; IGLÉZIAS, J. C. R.; PÊGO-FERNANDES P. M.; AULER JÚNIOR, J. O. C.; VERGINELLI, G.; JATENE, A. D. - Hemodynamic evaluation of myocardial protection by blood and acellular cardioplegic solutions: an experimental study. Rev. Bras. Cir. Cardiovasc., 5(3): 168$178,1990$.

ABSTRACT: This investigation was performed in order to evaluate the degree of myocardial protection in a group of 20 dogs. They were submitted to myocardial anoxia by cross-clamping of the ascending aorta for sixty minutes under extracorporeal circulation, followed by reperfusion for 120 minutes. Hemodynamic changes of the myocardium were studied, and the protection to anoxia offered by cardioplegic solutions and hypothermia was evaluated. The animals were divided in four groups with five dogs in each. In the first and second group, called control-group, myocardial anoxia was induced in normothermia and hypothermia $\left(28^{\circ} \mathrm{C}\right)$. In the third and fourth groups, besides the $28^{\circ} \mathrm{C}$ systemic hypothermia, coronary infusions of cardioplegic solutions with and without blood were performed. In each group of dogs a significant lowering of cardiac output was observed at $\mathbf{3 0}$ minutes of myocardial reperfusion. Followed 120 minutes, group III and IV dogs showed cardiac output recovevy, and the lack of elevation in left ventricular end-diastolic pressure, indicating preservation of its mechanical function. This was not observed in other animals, in which cardioplegia was not used. Both cardioplegic solutions used protect efficiently the myocardial cells against anoxia in functional aspects. It was not possible, however, to demonstrate any superiority of the blood solution over an acellular solution.

DESCRIPTORS: myocardial protection, cardioplegia. 
DALLAN, L. A.; OLIVEIRA, S. A.; IGLÉZIAS, J. C. R.; PÊGO-FERNANDES, P. M.; AULER JÚNIOR, J. O. C.; VERGINELLI, G.; JATENE, A. D. - Solução cardioplégica sangüínea versus acelular: avaliaçāo hemodinâmica através de estudo experimental em cäes. Rev. Bras. Cir. Cardiovasc., 5(3): 168-178, 1990.

\section{REFERÊNCIAS BIBLIOGRÁFICAS}

1 BRAILE, D. M.; BILAQUI, A.; ANACLETO, J. C.; ARAÚJO, J. D.; BELLINI, A. J.; GARZON, S. A.; GREGO, O. T.; ARDITO, R. V.; AYOUB, J. C.; BAUCIA, J. A.; WICHTENDAHL, R. F.; KURODA, G. Y.; LORGA, A. M. - Proteção miocárdica por cardioplegia. Arq. Bras. Cardiol., 33(Supl. 1): 199-210, 1979. B. Saunders Company, 1980. p. 434-448.

BRETSCHNEIDER, H. J.; HŪBNER, G.; KNOLL, D.; LOHR, B.; NORDBECK, H.; SPIECKERMANN, P. G. - Myocardial resistance and tolerance to ischemia: physiological and biochemical basis. J. Cardiovasc. Surg. (Torino), 16: 241-260, 1975.

DALLAN, L. A.; HIGUCHI, M. L.; LOPES, E.; BELLOTTI, G.; ARMELIN, E.; PILEGGI, F.; VERGINELLI, G.; JATENE, A. D.; SESSO, A. - Análise das faces de criofratura e cortes ultrafinos do sarcolema de cardiócitos e plasmalema do endotélio capilar do miocárdio infartado do cảo. Arq. Bras. Cardiol., 43(Supl. 1): 33, 1984 (Resumo).

FOLLETTE, D. M.; MULDER, D. G.; MALONEY Jr., J. V.; BUCKBERG, G. D. - Advantages of blood cardioplegia over continuous coronary perfusion or intermittent ischemia: experimental and clinical study. J. Thorac. Cardiovasc. Surg., 76: 604-619, 1978.

6 GAY Jr., W. A. \& EBERT, P. A. - Functional, metabolic and morphologic effects of potassium - induced cardioplegia. Surgery, 74: 284-290, 1973.

GOMES, O. M.; MORAES, N. L. B.; FIORELLI, A. I.; BRUM, J. M.; RIBEIRO, M. P.; CALONGE, H. C.; ARMELIN, E.; BITTENCOURT, D.; ZERBINI, E. J. Parada cardíaca anóxica. Três horas de proteçāo: nova soluçāo cardioplégica. Arq. Bras. Cardiol, 36: 8-12, 1981.

GROSSMAN, W.; BRAUNWALD, E.; MANN, T.; McLAURIN, L. P.; GREEN, L. H. - Contractile state of the left ventricle in man as evaluated from end systolic pressure - volume relations. Circulation, 56: 845-851, 1977.

HEARSE, D. J.; STEWART, D. A.; BRAIMBRIDGE, M.
V. - Cellular protection during myocardial ischemia: the development and characterization of a procedure for the induction of reversible ischemic arrest. Circulation, 54: 193-202, 1976.

KARLINER, J. S.; PETERSON, K. L.; ROSS Jr., J. Left ventricular myocardial mechanics: systolic and diastolic function. In: Cardiac catherization and angiography. Philadelphia, Lea \& Febiger, 1980. p. 245265.

KIRSCH U.; RODEWALD, G.; KALMAR, P. - Induced ischemia arrest. J. Thorac. Cardiovasc. Surg., 63: 121-130, 1972.

2 MELROSE, D. G.; DREYER, B.; BENTALL, H. H.; BAKER, J. B. - Eletive cardiac arrest, Lancet, 2: 21-22, 1955.

3 MURAD, H.; QUEIROZ, A. D.; MATTOS, S. C.; PIEDADE, M. M.; ALBUQUERQUE, P. C.; MURAD, L. H.; PEREIRA, G. C.; BASTOS, E. S.; JAZBIK, A. P. Proteçāo miocárdica por cardioplegia sangūínea durante circulaçāo extracorpórea: avaliaçāo ultra-estrutural miocárdica no cão. Arq. Bras. Cardiol., 41: 231-239, 1983.

4 NELSON, R. L.; FEY, K. H.; FOLLETTE, D. M.; LIVESAY, J. J.; De LAND, E. C.; MALONEY Jr., J. V.; BUCKBERG, G. D. - Intermittent infusion of cardioplegic solution during aortic cross-clamping. Surg. Forum, 27: 241243, 1977.

5 ROE, B. B.; HUTCHINSON, J. C.; FISHMAN, N. H.; ULLYOT, D. J.; SMITH, D. L. - Myocardial protection with cold, ischemic, potassium-induced cardioplegia. J. Thorac. Cardiovasc. Surg., 73: 366374, 1977.

6 S. A. S. INSTITUTE INC. - S. A. S. urser's guide statistics. New York, Edition Cary, 1982.

TIMM, N. H. - Multivariate analysis with applications in education and psychology. Monterey, Brooks Cole, 1975.

YANG, S. S.; BENTIVOGLIO, L. G.; MARANHĀO, V.; GOLDBERG, H. - From cardiac catheterization data to hemodynamic parameters. Philadelphia, F. A. Davis Company, 1978. p. 244-249. 